\title{
Experiencias positivas y negativas en relaciones de pareja de jóvenes y adolescentes
}

\author{
Positive and Negative Experiences in Couple Relationships of \\ Young People and Adolescents
}

\author{
Ángel Hernando-Gómez, Pablo Maraver-López, \& María Pazos-Gómez \\ Universidad de Huelva, Huelva, España
}

\begin{abstract}
Resumen: El objetivo de este estudio es conocer las experiencias positivas y negativas que una amplia muestra de adolescentes y jóvenes reconocen haber vivido en sus relaciones de pareja. Para ello, mediante una metodología descriptiva utilizando encuestas, se analizan estas experiencias de acuerdo al sexo, la edad y el tiempo de relación. La muestra es de 912 adolescentes y jóvenes con edades comprendidas entre los 14 y 29 años, que respondieron a un cuestionario sobre experiencias vividas en pareja y un autoinforme sobre conductas psicopatológicas en adolescentes. Los resultados más significativos destacan la existencia de una mayoría de mujeres jóvenes y adolescentes frente a hombres que informan sobre sus experiencias en relaciones de pareja, destacando como positivas las vividas en los primeros momentos de la relación (salidas iniciales, primeras caricias, etc.); y como negativas, las discusiones, peleas y los celos. Ante los resultados obtenidos, se pone de manifiesto la necesidad de intervenir con el colectivo de jóvenes y adolescentes desde la prevención, tratando de potenciar las experiencias positivas en las relaciones de pareja y de minimizar las experiencias negativas.
\end{abstract}

Palabras clave: relaciones de pareja, adolescencia, juventud, prevención.
Abstract: This study aims to understand the positive and negative experiences that a large sample of adolescents and young people recognize have lived in their relationships. To do this, these experiences where analyzed according to sex, age and how long the relationships last. The sample is 912 adolescents and young people aged between 14 and 29 years, who answered a questionnaire about experiences, lived in couples and a self-report on psychopathological behaviors in adolescents. The most significant outcomes concern the existence of a majority of girls versus boys who report those experiences, highlighting as positive those experienced in the early stages of the relationship (such as first meetings and caresses), and as negative, the disputes, fights and jealousy. Given the results, it is evident the need to work with the group of young people and adolescents from prevention, trying to enhance the positive experiences in relationships and minimize the negative ones.

Keywords: relationships, adolescence, youth, prevention.

Contacto: A. Hernando-Gómez. Departamento de Psicología Social, Evolutiva y de la Educación. Universidad de Huelva. Campus del Carmen S/N. 21071, Huelva, España. Correo electrónico: angel.hernando@dpsi.uhu.es

Cómo citar: Hernando-Gómez, A., Maraver-López, P., \& Pazos-Gómez, M. (2016). Experiencias positivas y negativas en relaciones de pareja de jóvenes y adolescentes. Revista de Psicología, 25(2), 1-19.

http://dx.doi.org/10.5354/0719-0581.2016.44745 


\section{Introducción}

En las relaciones de pareja de jóvenes y adolescentes ocurren multitud de experiencias positivas y negativas. Dentro de estas experiencias, se encuentran ciertas conductas violentas que se extienden, al igual que en las relaciones de pareja de personas adultas, en un continuo que va desde el abuso verbal y emocional, hasta la agresión sexual y el asesinato. Por desgracia, las investigaciones realizadas en los últimos años no han detectado un descenso en este tipo de conductas (Corral \& Calvete, 2006; Fernández-Fuertes, Orgaz, \& Fuertes, 2011; Fernández-Fuertes, Fuertes, \& Pulido, 2006; García-Díaz et al., 2013; González-Ortega, Echeburúa, \& De Corral, 2008; Hernando-Gómez, 2007; Muñoz-Rivas, Graña, O’Leary, \& González, 2009; O’Leary, Slep, Avery-Leaf, \& Cascardi, 2008; Sears, Byers, \& Price, 2007; Soldevila, Domínguez, Giordano, Fuentes, \& Consolini, 2012; Vizcarra, Poo, \& Donoso, 2013); por el contrario, las altas prevalencias se mantienen llegando incluso a aumentar en algunos tipos de violencia. Ya nadie discute el hecho de que las experiencias negativas en pareja necesariamente tengan que comenzar después del matrimonio. De hecho, se ha constatado que, cada vez más, estas se dan en las primeras etapas de las relaciones de pareja o al comienzo de la convivencia (Gorrotxategi \& De Haro, 1999).

También se ha observado que las relaciones de pareja comienzan cada vez a una edad más temprana (Price \& Byers, 1999). Se entiende por estas “una relación diádica que involucra interacción social y actividades conjuntas con la implícita o explícita intención de continuar la relación hasta que una de las partes la termine o se establezca otro tipo de relación como la cohabitación o matrimonio" (Straus, 2004, p.
792). Se ha encontrado que diversas formas de control exagerado, que han sido pormenorizadamente enumeradas (Ferreira, 1992)-como exigir explicaciones por todo y pretender conocer hasta su pensamiento más recóndito, querer saber con todo lujo de detalles dónde va y dónde estuvo, formular prohibiciones o amenazas respecto a los estudios, el trabajo, las costumbres, etc.- comienzan a aparecer de forma temprana $y$, poco a poco, este tipo de conductas abusivas se van haciendo cada vez más frecuentes y extremas.

Son muchas las definiciones que se pueden encontrar en la literatura sobre dating violence o violencia entre parejas jóvenes (Close, 2005; Wolfe, Wekerle, Gough, \& Reitzel-Jaffe, 1996). Las primeras definiciones solo contemplaban como conductas violentas las agresiones físicas, tal y como podemos ver, por ejemplo, en la planteada por Sugarman y Hotaling (1989): "el uso o amenaza de fuerza física o contención física llevada a cabo con la intención de causar dolor o daño al otro" (p. 4).

Con posterioridad, se fueron introduciendo en estas las agresiones verbales, psicológicas, sexuales, etc. Hasta llegar a las recientes definiciones que ya contemplan los distintos tipos de agresión. Destaca entre todas ellas, por considerar que todo tipo de abuso es perjudicial y que, por tanto, debe ser tenido en cuenta, la planteada por la Public Health Agency of Canada que la define como "todo ataque intencional de tipo sexual, físico o psíquico, de un miembro de la pareja contra el otro en una relación de pareja" (Health Canada, 1995).

Este es un fenómeno que puede afectar a cualquiera con independencia de la edad, raza, orientación sexual, estatus socioeconómico o lugar de residencia. Los comportamientos negativos hacia la pareja pueden 
tomar muchas formas, incluyendo el abuso emocional, psicológico, físico o sexual, que pueden coexistir, o manifestarse de una manera aislada; a su vez, la violencia puede presentarse tanto si ha transcurrido mucho tiempo como poco tiempo desde el comienzo de la relación (Eaton et al., 2006). En la definición realizada de violencia de género por el mismo autor, se le considera como la violencia física, sexual o psicológica/emocional que ocurre dentro de una relación de pareja, así como los actos de persecución o acoso (CDC, 2016). Además, Eaton et al. (2010) especifica que este tipo de conductas pueden darse por parte de una pareja actual o pasada, en persona o a través de diferentes medios electrónicos, tal y como se indica en HernandoGómez (2007), quien detecta que a las formas de abuso descritas en Ferreira (1992) hay que añadir las realizadas por medios electrónicos y, actualmente, todas las relacionadas con la ciberviolencia (Torres, Robles, \& De Marco, 2013).

Las experiencias negativas con signos de violencia en las relaciones de pareja se reconocen en la actualidad como un problema social. Este hecho ha supuesto un avance social en el estudio y diseño de programas de intervención sobre el fenómeno, ya que ha significado que, tanto desde la administración pública como desde el mundo académico, esté recibiendo una gran atención, la que se ha traducido en recientes investigaciones e intervenciones sobre las actitudes y comportamientos violentos en adolescentes. A pesar de la atención, las líneas de investigación de este problema se encuentran todavía en sus inicios. Una explicación posible a este hecho está directamente relacionada con la dificultad que tienen adolescentes y jóvenes para reconocer que son víctimas del maltrato, por interpretar erróneamente las conductas violentas como expresiones de amor (García Díaz et al., 2013; González
Méndez \& Santana Hernández, 2001; Vizcarra et al., 2013).

Otros aspectos que inciden en la invisibilización de este fenómeno son: la idealización que adolescentes y jóvenes realizan de las conductas violentas, con base en la noción del amor romántico, la justificación de las conductas, y el hecho de quitarle importancia a comportamientos violentos como los celos y el control obsesivo, entre otros (Soldevila et al., 2012). Añadido a este no reconocimiento y error en la explicación, otra dificultad para investigar e intervenir en esta temática es la ocultación que hace la víctima de la violencia que se ejerce sobre ella. Distintos estudios han encontrado que la mayoría de personas que sufren experiencias negativas en pareja lo mantienen en secreto (Ashley \& Foshee, 2005; Molidor \& Tolman, 1998), y cuando se lo comentan a alguien, en general lo hacen a sus iguales $y$, en menor medida, a sus familiares. Esta preferencia viene explicada por la creencia de que sus iguales los van a entender mejor (Ocampo, Shelley, \& Jaycox, 2007; Vizcarra \& Poo, 2011; Weisz, Tolman, Callahan, Saunders, \& Black, 2007). A esta ocultación también ayuda el hecho de que los adolescentes piensan que acudir a los recursos legales no les va a ayudar, y que si piden ayuda al sistema legal -usualmente desconocen los procedimientos legales, qué hacer y adónde acudir - no les creerán, se perderá la confidencialidad (Ocampo et al., 2007) o se enterarán sus padres.

En el ámbito internacional, a partir de la década de 1980 con el trabajo pionero realizado por Makepeace (1981), la violencia en las relaciones de pareja cobró gran importancia tanto a nivel de investigación como en lo relativo al diseño y realización de programas de prevención en el entorno escolar. Una gran parte de las investigaciones realizadas en los años transcurridos ha 
tenido como objetivo establecer la prevalencia de este tipo de violencia.

Estudios realizados en Estados Unidos reflejan prevalencias de experiencias negativas en relaciones de pareja jóvenes y adolescentes muy diversas, oscilando entre el 9\% (Roscoe \& Callahan, 1985) y el 57\% (Avery-Leaf, Cascardi, O’Leary, \& Cano, 1997), o entre el $25 \%$ y el $45 \%$ (Straus \& Ramírez, 2007). De la misma forma, Cornelius y Resseguie (2007) exponen datos que apuntan a una posible explicación de esta alta variabilidad, la diferencia de medida, ya que cuando en la investigación se incluyen conductas agresivas de tipo verbal, la prevalencia sube hasta el $88 \%$.

De los estudios sobre experiencias negativas en adolescentes y jóvenes destacan las aportaciones realizadas por González Méndez y Santana Hernández (2001), quienes informan que el $7,5 \%$ de los hombres y el $7,1 \%$ de las mujeres reconocen que en una o más ocasiones han pegado o empujado a su pareja. También resultan llamativos los resultados descritos por Muñoz-Rivas, Graña, O’Leary, \& González (2007), que hallaron que en torno al $90 \%$ de los estudiantes de la muestra, de 16 a 20 años, manifestaron que en algún momento habían agredido verbalmente a su pareja, mientras que el $40 \%$ expuso haber ejercido la violencia física. A estas investigaciones debemos añadir otras más recientes cuyos resultados muestran que este fenómeno está muy presente en los adolescentes y jóvenes, ya que en ellos se constata la presencia de conductas violentas en las relaciones de pareja como forma de resolver los conflictos que surgen en su interior (Díaz-Aguado, Martínez-Arias, \& Martín-Babarro, 2013; González-Ortega et al., 2008). En concreto, en el estudio realizado por Díaz-Aguado y Carvajal (2011) se muestra que el 13\% de los hombres reconoce haber ejercido o intentado situaciones de maltrato y que el 9,2\% de las mujeres ha sufrido en alguna ocasión maltrato físico o psicológico por alguna de sus parejas.

A nivel de investigación, existe una falta de acuerdo sobre las metodologías a seguir para investigar el fenómeno, así como qué muestras se deben elegir y qué tipos de análisis se deben realizar. Esto ha dado lugar a la alta diferencia de las prevalencias encontradas. Aun así, existe un acuerdo, entre los resultados obtenidos en los estudios revisados sobre la temática, en que las diversas investigaciones muestran que tanto la perpetración como la victimización de agresiones son muy frecuentes (Kaukinen, Gover, \& Hartman, 2012). Dichas agresiones suelen ser bidireccionales y son menos graves en comparación con la violencia ejercida en relaciones de pareja donde existe convivencia y en que los miembros son de mayor edad. Específicamente, la violencia ejercida en parejas jóvenes y adolescentes es de dos a tres veces mayor que la ejercida en parejas adultas, aunque sus consecuencias no suelen ser tan graves (Karakurt \& Cumbie, 2012; Menesini \& Nocentini, 2008; Ortega, Ortega Rivera, \& Sánchez, 2008).

Este aspecto es muy importante y debe ser tenido en cuenta, entre otras acciones, para el diseño de programas de prevención. Se hace necesario, romper la dicotomía varón-agresor, mujer-víctima. Este enfoque, que sí es aplicable a edades adultas, no responde a los índices de prevalencia que encontramos en edades tempranas, sino que aparece una cierta paridad, que varía en función de las conductas agresivas estudiadas, en los comportamientos agresivos de hombres y mujeres en las relaciones de pareja, lo que no implica que la vivencia y las consecuencias de esta violencia sufrida sea igual para ambos sexos (Gomez, Speizer, \& Moracco, 2011; O’Leary \& Slep, 2012). 
Los datos obtenidos en jóvenes y adolescentes, con respecto a diferencias de sexo, no permiten todavía concluir evidencia empírica con respecto al sexo de los agresores, ya que existen diferencias entre los distintos estudios revisados. Al respecto, en algunas investigaciones son los hombres los que con más frecuencia son señalados como agresores (Makepeace, 1981; Tontodonato \& Crew, 1992), mientras que en otras son las mujeres (Fernández-Fuertes \& Fuertes, 2010; González-Ortega et al., 2008); otros indican la existencia de una alta bidireccionalidad (FernándezGonzález, 2013; Weisz et al., 2007).

Diversos autores han expuesto el hecho de que los hombres tienden más a legitimar las conductas violentas como respuesta y les restan importancia, mientras que las mujeres realizan una sobrevaloración de sus actos y se sienten culpables por ello (González-Ortega et al., 2008). Algunos resultados han puesto de relieve que los hombres suelen rechazar menos la violencia y la justifican más que las mujeres (Garaigordobil, Aliri, \& Martínez-Valderrey, 2013); una explicación posible es el alto grado con el que estos jóvenes asimilan las actitudes y creencias machistas de la sociedad (Arenas García, 2013).

Es de resaltar que en parejas de jóvenes y adolescentes la permanencia en las relaciones violentas presenta particularidades con respecto a las adultas. El hecho de continuar en la relación se puede explicar por la inmadurez emocional, la intensidad del sentimiento, las creencias en estereotipos de género sobre roles y modelos sexistas, y los mitos del amor romántico, ya que en edades tempranas no existe dependencia económica, hijos o presiones de tipo familiar o social (González-Ortega et al., 2008; Hernando-Gómez, 2007).
A pesar de las altas prevalencias encontradas en los estudios revisados, el fenómeno en cuestión sigue dándose -con diferencias entre los distintos países- como algo invisible y minimizado a nivel social. Los actos violentos están tan arraigados y presentes en la sociedad que, aunque han existido siempre, cuesta identificarlos; ante eso, lo nuevo es verlo como violencia y no aceptarla (Alberdi \& Rojas, 2005). La normalización de estos comportamientos en la adolescencia es posiblemente mayor que en otras edades, puesto que ellos y ellas, si bien son capaces de describir la violencia, conocen casos de violencia de género y pueden identificarla sobre el papel, en general, creen que se trata de algo que solo les ocurre a mujeres mayores que ya están casadas (Hernando-Gómez, 2007). Además, se da la circunstancia de que determinados comportamientos, que están en la base y en el inicio del problema, como los celos y el control exagerado, para muchos adolescentes son síntomas de amor y preocupación por la pareja, y no lo ven como el posible germen del problema (García Díaz et al., 2013; González Méndez \& Santana Hernández, 2001; Soldevila et al., 2012; Vizcarra et al., 2013). Las razones para disculpar las conductas violentas están presentes en nuestros jóvenes y ellos siguen los mismos mitos y falsas creencias sobre el tema, tal y como corresponde a los roles sociales de la comunidad en la que están insertos.

De esta manera, nos encontramos frente a un problema complejo, ya que es multidimensional y está influenciado por factores relativos a la persona que maltrata, a la víctima, y al contexto familiar y sociocultural donde se produce. Las investigaciones al respecto han incrementado el conocimiento sobre determinados factores de riesgo que pueden hacer a adolescentes y 
jóvenes más vulnerables a la victimización y a la perpetración (Anderson \& Whiston, 2005; Díaz-Aguado \& Martínez Arias, 2002; Follingstad, Bradley, Laughlin, \& Burke, 1999; Foshee et al., 2011; González-Ortega et al., 2008; Greytak, 2003; Meras Lliebre, 2003; Sugarman \& Hotaling, 1989). Estos factores de riesgo son atributos que están asociados con un incremento de la probabilidad de recibir conductas violentas o perpetrarlas. Aquellos que están relacionados con los comportamientos violentos en las relaciones de pareja pueden ser individuales, relacionales y contextuales; y la compleja interacción entre estos factores crea las circunstancias en las cuales se pueden producir actos de violencia en las relaciones de pareja entre adolescentes.

Con respecto a uno de los principales componentes presentes en las relaciones de pareja entre adolescentes, los celos, hay estudios que los relacionan con la presencia de inestabilidad afectiva, trato parental negativo y síntomas de trauma; además encuentran una capacidad predictiva entre el uso de la violencia y distintas variables como las estrategias de resolución de conflictos y la inteligencia emocional de la persona que agrede (Perles Novas, San Martín García, Canto Ortiz, \& Moreno Jiménez, 2011).

Aunque existen pocos estudios que contemplen dentro de sus objetivos investigar sobre cómo influyen las variables psicopatológicas en las relaciones de pareja entre adolescentes, sí se han estudiado estas variables con respecto a la violencia entre iguales, de donde se ha observado que en ambos tipos de violencia se da una opresión reiterada entre personas en las que existe un desequilibrio de poder (Pazos Gómez, Oliva Delgado, \& Hernando-Gómez, 2014). A partir de estos estudios se puede decir que existiría una agresión abierta, tradicionalmente atribuida a hombres, asociada a problemas externalizantes como la impulsividad o las conductas desafiantes. Por otra parte, se daría una agresividad relacional, más frecuente entre las mujeres y vinculada a problemas internalizantes como la tristeza, la ansiedad o las quejas somáticas (Crick \& Grotpeter, 1995). Es probable que estos patrones de conducta agresiva se presenten también cuando la violencia es ejercida sobre la pareja.

Vemos entonces que distintas investigaciones han identificado una serie de factores de riesgo para la violencia en las relaciones de pareja entre adolescentes, tanto para la perpetración como para la victimización. Ciertamente, estos factores deben ser tenidos en cuenta a la hora de diseñar acciones preventivas.

Los datos que se presentan en este trabajo forman parte de una investigación más amplia sobre las situaciones de dominio y posesión en las relaciones de pareja de adolescentes y jóvenes. En concreto, en este estudio se abordan los resultados obtenidos acerca de las experiencias vividas en pareja, positivas y negativas, descritas por las personas participantes. En esa línea, el objetivo general es conocer las situaciones tanto positivas como negativas que la experiencia en pareja les ha aportado a los componentes de la muestra. Para ello, en concreto, se abordan los siguientes objetivos específicos: 1) analizar las experiencias positivas vividas en parejas de jóvenes y adolescentes, 2) analizar las experiencias negativas experimentadas en parejas de jóvenes y adolescentes, 3) analizar las diferencias de sexo con relación al tipo de experiencias vividas en pareja, y 4) analizar las diferencias en experiencias vividas en la pareja, en función de las variables tiempo en pareja, edad y 
presencia de problemáticas internalizantes y externalizantes.

\section{Método}

\section{Diseño}

Con la intención de dar respuesta a los objetivos planteados con anterioridad, esta investigación sigue una metodología descriptiva de encuestas. Se analizan las experiencias positivas y negativas percibidas por los participantes de acuerdo al sexo, la edad y el tiempo de relación, en una muestra de adolescentes y jóvenes que respondieron a un cuestionario sobre experiencias vividas en pareja y a una escala sobre conductas psicopatológicas en adolescentes (Achenbach, 1991; Lemos Giráldez, Vallejo Seco, \& Sandoval Mena, 2002).

\section{Participantes}

La muestra está compuesta por un total de 912 personas con edades comprendidas entre los 14 y 29 años de edad que cursan educación secundaria obligatoria, bachillerato y ciclos formativos en diversos institutos de educación secundaria obligatoria de Huelva capital y provincia (España). Este tramo de edad tan amplio permite incluir a la totalidad de las personas adolescentes y jóvenes que se encuentran cursando sus estudios en el momento de la investigación, ya que están incorporadas dentro de la escala de edades en la adolescencia y juventud propuesta por la Organización Mundial de la Salud (OMS) en el año 2000, que considera la juventud hasta los 32 años. El criterio de selección de la muestra sigue un muestreo de juicios de casos típicos: 12 centros educativos públicos que resultan accesibles. La distribución de dicha muestra por sexo es la siguiente: el $63,1 \%$ de los sujetos son mujeres y el $36,3 \%$ restante pertenece al sexo masculino. La media de edad es de 16,26 $(D T=2,25)$ años siendo mayoritario el grupo de sujetos de edades comprendidas entre los 15 a 17 años de edad.

De los cuatro cursos que componen la etapa de educación secundaria obligatoria, los sujetos pertenecientes al primer curso no han sido analizados en esta investigación, debido a que en dichas edades (12-13 años) las relaciones de pareja son menores y de duración esporádica, de tal forma que las experiencias referidas en pareja aún no manifiestan señales dignas de consideración. Asimismo, se ha seleccionado solo al alumnado que, en el momento de la recogida de datos, mantenía o había mantenido en el último año una relación de pareja con una duración mínima de un mes. En la tabla 1 se puede observar el número de personas (un total de 912) que se encontraban manteniendo una relación en el momento de la prueba y la duración de dicha relación.

Tabla 1

Tiempo de relación en pareja

\begin{tabular}{lcc}
\hline \multicolumn{1}{c}{ Duración } & Frecuencia & Porcentaje \\
\hline Actualmente no & 265 & 29,9 \\
0 a 6 meses & 256 & 28,1 \\
6 a 12 meses & 123 & 13,5 \\
1 a 2 años & 136 & 14,9 \\
2 a 3 años & 92 & 10,1 \\
Más de 3 años & 40 & 4,4 \\
\hline
\end{tabular}

\section{Instrumentos}

Datos sociodemográficos. Cuestionario constituido por ocho ítems de respuesta abierta, construidos ad hoc, donde se recogen: edad, sexo, nivel de estudio, lugar de residencia, zona de residencia, orientación sexual, tiempo y tipo de relación.

Cuestionario de experiencias vividas en pareja. Es un cuestionario construido ad 
hoc y compuesto por dos preguntas abiertas donde se solicita que reflejen aquellas experiencias positivas vividas en pareja, como también las de carácter negativo. De acuerdo con los principios de sistematicidad, objetividad y fiabilidad del análisis de contenido, se emplearon tres personas expertas codificadoras para la interpretación de los mensajes. En primer lugar se establecieron las categorías. Asimismo, se codificó por separado cada unidad de análisis, comparando posteriormente las interpretaciones y se comprobaron las discrepancias. A través de varias puestas en común, se llegó a un consenso para la codificación y se consiguió reducir al mínimo la distancia entre las discrepancias iniciales. La confiabilidad entre calificadores para el análisis final fue de, 91 .

Autoinforme para Jóvenes (YSR, por su nombre en inglés Youth Self Report) (Achenbach, 1991). Adaptación a la población española de Lemos Giráldez et al. (2002). Es un autoinforme de screening de conductas psicopatológicas en adolescentes. La escala está compuesta por 45 ítems tipo Likert en una escala de 0 (No es verdad) a 2 (Muy verdadero). Evalúa la psicopatología a través de distintos factores que quedan agrupados en dos manifestaciones: problemas externos y problemas internos. Se compone de siete subdimensiones centrales o comunes a ambos sexos: depresión/ansiedad, conducta delictiva, conducta agresiva, quejas somáticas, problemas de pensamiento, problemas de relación, y conductas de búsqueda de atención.

El coeficiente de fiabilidad alfa de Cronbach, para la Subescala de Problemas Internalizantes es de ,81, para la Subescala de Problemáticas Externalizantes, 93 y ,73 para la escala global. Sus evidencias de validez pueden encontrarse en Lemos et al. (2002).

\section{Procedimiento}

Conociéndose la función de enlace y coordinación que ejercen los orientadores y orientadoras de los centros educativos, el primer contacto se mantuvo con estos profesionales, quienes nos facilitaron el acceso tanto al equipo directivo como a los distintos profesores y profesoras cuyas clases se desarrollaban en las horas fijadas para la realización de las encuestas.

Todos los instrumentos se completaron individual y voluntariamente en el aula, previa autorización de las familias en el caso de los menores de edad. Se informó a los estudiantes, en una primera sesión, del carácter voluntario de su participación, así como del anonimato y confidencialidad de los datos recogidos y se repartió el documento de consentimiento informado. En los días siguientes se procedió a la cumplimentación de los instrumentos. Tanto el profesorado como las personas encuestadoras estuvieron presentes durante todo el proceso y agradecieron la participación de las personas en el estudio.

\section{Resultados}

Siguiendo el principal objetivo del estudio, se presentan los resultados obtenidos acerca de las experiencias vividas en pareja, positivas y negativas, descritas por los sujetos encuestados. En primer lugar, se da respuesta al objetivo específico 1: analizar las experiencias positivas vividas en parejas de jóvenes y adolescentes. Al comparar las frecuencias obtenidas de las distintas experiencias positivas remitidas en pareja se puede observar que la experiencia positiva más frecuentemente indicada por los sujetos fue "salir juntos", con un $26 \%$, seguida de la categoría "primer beso y primeros momentos juntos", con un $22 \%$. 
En segundo lugar, se aborda el objetivo específico 2: analizar las experiencias negativas experimentadas en parejas de jóvenes y adolescentes. Respecto a las experiencias negativas, las experiencias vividas en pareja más frecuentemente remitidas por los sujetos fueron las discusiones y peleas físicas (43\%), seguida de la ruptura (20\%), de otras experiencias (14\%) y los celos (10\%). El $1 \%$ de los sujetos consultados remitieron como experiencia negativa haberse visto obligados a mantener relaciones sexuales con la pareja.

En tercer lugar, respecto al objetivo 3 analizar las diferencias de sexo con relación al tipo de experiencias vividas en pareja-, tanto en las experiencias positivas como en las negativas, la prueba Chi cuadrado de Pearson indicó la existencia de diferencias estadísticamente significativas en función del sexo tanto en experiencias positivas, $\chi^{2}(7, n=968)=64,721, p<, 05$, como negativas $\chi^{2}(6, n=883)=15,609, p$ $<, 05$. Así, la proporción de mujeres que remiten haber vivido experiencias positivas es mayor que la de los hombres en todas las categorías, a excepción de la categoría de "relaciones sexuales", donde el porcentaje es superior en hombres (52\% en hombres frente a un $48 \%$ en mujeres). La categoría "todo buenas experiencias" es señalada por un $85,6 \%$ de mujeres frente a un $14,4 \%$ en hombres, o la categoría "muestras de apoyo, cariño, regalos..." con un $86,1 \%$ en mujeres frente a un $13,9 \%$ en hombres, entre otras (tabla 2).

Respecto a las experiencias negativas vividas en pareja, el porcentaje en mujeres es superior al de los hombres en la totalidad de categorías contempladas. La categoría "discusión y peleas físicas" es señalada por un $70,4 \%$ en mujeres, frente a un $22,6 \%$ en hombres; y los "celos" con un $70,2 \%$ en mujeres, frente a un $29,8 \%$ en hombres.
En cuarto lugar, se presentan los resultados que responden al objetivo 4 sobre las diferencias en experiencias negativas vividas en la pareja, en función de las variables "tiempo en pareja", "edad" y "presencia de problemáticas internalizantes y externalizantes". La prueba Chi cuadrado de Pearson reveló diferencias estadísticamente significativas $\chi^{2}(18, n=$ $493)=32,323, p<, 05$ en función del tiempo en pareja, categorizada en cuatro grupos (No tengo pareja, de 0 a 12 meses, de 12 a 36 meses, más de 36 meses). Tal y como se puede observar en la tabla 2 , la franja de 12 a 36 meses obtuvo porcentajes superiores al resto de franjas de tiempo en todas las categorías, con excepción de la categoría "celos", donde obtuvo un porcentaje superior la franja de tiempo de 0 a 12 meses. La categoría "obligación de mantener relaciones sexuales" estuvo presente en todas las franjas de tiempo en pareja a excepción de la franja "más de 36 meses", donde no se registró ningún caso. Asimismo, en las categorías "infidelidad", "discusiones/peleas físicas" y "malentendido/terceras personas" se registró una mayor frecuencia, encontrándose dentro de la franja de tiempo en pareja de 12 a 36 meses, con un 41,2\%, 49,1\% y $60 \%$ respectivamente.

Respecto a las diferencias en función de la edad, la prueba de Chi cuadrado reveló diferencias estadísticamente significativas en las experiencias negativas vividas en pareja $\chi^{2}(12, n=899)=34,792, p<$ ,05. Concretamente, el rango de 16 a 18 años fue en donde se manifestaron más experiencias negativas vivenciadas en la pareja, siendo las más frecuentemente remitidas las categorías de "obligación de mantener relaciones sexuales", "malentendido/terceras personas" y "ruptura", con un $85,7 \%, 75,9 \%$ y $72,9 \%$ respectivamente. 
Tabla 2

Experiencias positivas y negativas de acuerdo a diferentes variables en la relación

\begin{tabular}{|c|c|c|c|c|c|c|c|c|c|c|}
\hline \multirow[b]{2}{*}{ Categoría } & \multicolumn{2}{|c|}{ Sexo } & \multicolumn{4}{|c|}{ Tiempo de relación } & \multicolumn{3}{|c|}{ Edad } & \multirow[t]{2}{*}{ Total } \\
\hline & Hombre & Mujer & $\begin{array}{l}\text { No } \\
\text { tengo }\end{array}$ & $\begin{array}{c}0-12 \\
\text { meses }\end{array}$ & $\begin{array}{l}12-36 \\
\text { meses }\end{array}$ & $\begin{array}{l}>36 \\
\text { meses }\end{array}$ & $\begin{array}{c}12-15 \\
\text { años }\end{array}$ & $\begin{array}{l}16-18 \\
\text { años }\end{array}$ & $\begin{array}{c}19-29 \\
\text { años }\end{array}$ & \\
\hline \multicolumn{11}{|l|}{ Experiencias positivas } \\
\hline $\begin{array}{l}\text { Todo buenas expe- } \\
\text { riencias }\end{array}$ & $14,4 \%$ & $85,6 \%$ & $7,4 \%$ & $11,5 \%$ & $13,2 \%$ & $25,4 \%$ & $4,6 \%$ & $14 \%$ & $18,6 \%$ & $13 \%$ \\
\hline Salir a divertirse & $26,5 \%$ & $73,5 \%$ & $0 \%$ & $4,1 \%$ & $6,1 \%$ & $0 \%$ & $4,6 \%$ & $3,4 \%$ & $4,7 \%$ & $4 \%$ \\
\hline $\begin{array}{l}\text { Muestras de apoyo, } \\
\text { cariño, regalos... }\end{array}$ & $13,9 \%$ & $86,1 \%$ & $11,4 \%$ & $8,6 \%$ & $12,6 \%$ & $7,9 \%$ & $10,7 \%$ & $10,4 \%$ & $9,3 \%$ & $10 \%$ \\
\hline Relaciones sexuales & $52 \%$ & $48 \%$ & $10,2 \%$ & $9,9 \%$ & $12,6 \%$ & $33,3 \%$ & $7,1 \%$ & $14,3 \%$ & $11,6 \%$ & $13 \%$ \\
\hline Reconciliación & $17,5 \%$ & $82,5 \%$ & $6,8 \%$ & $3,9 \%$ & $7,4 \%$ & $11,1 \%$ & $5,1 \%$ & $6,1 \%$ & $7 \%$ & $6 \%$ \\
\hline $\begin{array}{l}\text { Primer beso y pri- } \\
\text { meros momentos }\end{array}$ & $32,2 \%$ & $67,8 \%$ & $21,7 \%$ & $28,6 \%$ & $16,2 \%$ & $3,2 \%$ & $34 \%$ & $20,4 \%$ & $11,6 \%$ & $22 \%$ \\
\hline Salir juntos & $29,9 \%$ & $70,1 \%$ & $34,2 \%$ & $28,9 \%$ & $22 \%$ & $19 \%$ & $25,6 \%$ & $25,9 \%$ & $27,9 \%$ & $26 \%$ \\
\hline Otras experiencias & $41,3 \%$ & $58,7 \%$ & $8 \%$ & $4,4 \%$ & $9,7 \%$ & $0 \%$ & $7,6 \%$ & $5,5 \%$ & $9,3 \%$ & $6 \%$ \\
\hline \multicolumn{11}{|l|}{ Experiencia negativas } \\
\hline Infidelidad & $31,3 \%$ & $68,7 \%$ & $7,8 \%$ & $35,3 \%$ & $41,2 \%$ & $15,7 \%$ & $18,1 \%$ & $67,5 \%$ & $14,5 \%$ & $9 \%$ \\
\hline $\begin{array}{l}\text { Discusiones/peleas } \\
\text { físicas }\end{array}$ & $22,6 \%$ & $77,4 \%$ & $7,4 \%$ & $35,7 \%$ & $49,1 \%$ & $7,7 \%$ & $17,4 \%$ & $65,0 \%$ & $17,6 \%$ & $43 \%$ \\
\hline Ruptura & $33,5 \%$ & $66,5 \%$ & $17 \%$ & $33 \%$ & $36,8 \%$ & $13,2 \%$ & $23,8 \%$ & $72,9 \%$ & $3,3 \%$ & $20 \%$ \\
\hline Celos & $29,8 \%$ & $70,2 \%$ & $10,2 \%$ & $53,1 \%$ & $36,7 \%$ & $0 \%$ & $18,6 \%$ & $67,4 \%$ & $14,0 \%$ & $10 \%$ \\
\hline $\begin{array}{l}\text { Mal entendido/Ter- } \\
\text { ceras personas }\end{array}$ & $44,8 \%$ & $55,2 \%$ & $5 \%$ & $35 \%$ & $60 \%$ & $0 \%$ & $24,1 \%$ & $75,9 \%$ & $0 \%$ & $3 \%$ \\
\hline $\begin{array}{l}\text { Obligación de man- } \\
\text { tener relaciones }\end{array}$ & $42,9 \%$ & $57,1 \%$ & $28,6 \%$ & $28,6 \%$ & $42,9 \%$ & $0 \%$ & $14,3 \%$ & $85,7 \%$ & $0 \%$ & $1 \%$ \\
\hline Otras experiencias & $34,5 \%$ & $65,5 \%$ & $7,1 \%$ & $48,7 \%$ & $36,5 \%$ & $7,7 \%$ & $23,8 \%$ & $68,9 \%$ & $7,4 \%$ & $14 \%$ \\
\hline
\end{tabular}

Por último, respecto a los resultados encontrados entre la variable experiencias negativas vividas en pareja y la presencia de problemáticas internalizantes y externalizantes, solo se encontraron diferencias estadísticamente significativas en la dimensión conducta fóbico-ansiosa de la Subescala de Problemáticas Internalizantes $(F(6,515)$ $=2,462, p<, 05)$. Las personas que presentaron una puntuación elevada en esta dimensión remiten haber vivido más experiencias negativas en la pareja que el resto de sujetos que puntúan en otras dimensiones de la escala. Concretamente, como experiencias negativas vividas más frecuentemente se identifican "la infidelidad" $(M=1,80 ; D T=$ $0,42)$, "las discusiones/peleas físicas" $(M=$ $1,73 ; D T=0,41)$, "la ruptura" $(M=1,72 ; D T$ $=0,46) \mathrm{y}$ "mal entendidos/terceras personas" $(M=1,67 ; D T=0,32)$.

\section{Discusión y conclusiones}

Según los datos del presente estudio, las experiencias más señaladas por los jóvenes son las discusiones y peleas físicas (43\%), seguido de las rupturas (20\%) y otras experiencias (14\%). En el caso de las mujeres encuestadas, una experiencia negativa bastante señalada son los celos $(70,2 \%)$. A todo lo anterior se debería sumar que la violencia verbal y emocional es percibida por ambos sexos como el modo más usual en que hombres y mujeres cometen y sufren situaciones violentas en el seno de sus parejas.

Respecto a la presencia de experiencias negativas experimentadas en relación con la edad, se aprecia cómo en el inicio de la adolescencia (de 12 a 15 años) las princi- 
pales categorías mencionadas son la ruptura, y los malentendidos y terceras personas, con porcentajes de $24,1 \%$ y $23,8 \%$, respectivamente. El siguiente grupo de adolescentes investigados (de 16 a 18 años) manifiestan especialmente sentirse obligados a mantener relaciones sexuales, seguido de la ruptura y los celos, en un porcentaje mucho mayor $(85,7 \%, 72,9 \%$ y $67,4 \%$ respectivamente). Finalmente, los jóvenes de 19 a 29 años señalan principalmente las discusiones y peleas físicas, infidelidad y celos $(17,6 \%, 14,5 \%$ y $14 \%$ respectivamente). Estos resultados también han de ser tenidos en cuenta a la hora de crear dinámicas de trabajo en las diferentes etapas educativas a incidir, así como en el diseño de los programas a implementar en cada franja etaria.

Por otro lado, las parejas de menor trayectoria (0 a 12 meses) muestran un mayor grado de experiencias con celos $(53,1 \%)$, seguidos de los malentendidos, discusiones y peleas, obligación a mantener relaciones sexuales e infidelidad (60\%, 49, $1 \%$, $42,9 \%$ y $41,2 \%$ respectivamente) que cuando estas están más consolidadas (12 a 36 meses). En parejas más maduras (más de 36 meses) la posible ruptura $(13,2 \%)$ es una de las experiencias más mencionadas, mientras que la obligación a mantener relaciones sexuales pareciera ya no ser un problema para la pareja.

Estos datos coinciden con diversas investigaciones que respaldan el hecho de que las mujeres ejerzan un papel preponderante en los episodios de violencia de tipo verbal, emocional y física, de manera leve (Sears et al., 2007; Fernández-Fuertes \& Fuertes, 2010; Muñoz-Rivas et al., 2007), mientras que los hombres destacan en violencia sexual, principalmente (FernándezFuertes \& Fuertes, 2005; Ortega et al., 2008; Sears et al., 2007).
Una vez realizado un análisis pormenorizado de las respuestas ofrecidas por los integrantes de la muestra, llegamos a la conclusión de que el modo de concebir los momentos deseables -entendiéndolos como comportamientos positivos- y no deseables -entendiéndolos como comportamientos negativos- ofrecen un patrón similar de respuestas. En el primer caso, los jóvenes y adolescentes refieren los primeros acercamientos y besos, las muestras de apoyo y cariño, la posibilidad de pasar tiempo a solas y las relaciones sexuales, aunque en menor medida, como momentos deseables. En el polo opuesto encontramos las discusiones, los celos, la infidelidad o el momento de la ruptura, como situaciones negativas y problemáticas que bien pueden suponer el inicio de una situación violenta.

Conociendo esta realidad, se debiese intentar dirigir esfuerzos a influir en aquellas circunstancias que se mantienen más o menos constantes en los adolescentes y que condicionan el establecimiento de relaciones poco o nada saludables. Unido a lo anterior, se debiese incidir en la promoción de relaciones saludables, basándonos en un mayor grado de conocimiento de la violencia de parejas y los desencadenantes de los comportamientos violentos, tratando de dotar a hombres y mujeres de herramientas y habilidades suficientes como para ser capaces de hacer frente a estas situaciones. Para ello, los programas de prevención se constituyen como un instrumento fundamental, siempre y cuando estén adecuadamente diseñados, atendiendo a los destinatarios finales, contextualizando las sesiones de trabajo a su realidad y partiendo de sus experiencias e inquietudes en un clima de seguridad y confianza, que garantice la confidencialidad y voluntariedad de los participantes. En este sentido, los centros escolares ejercen una importante labor dentro de un continuo, en el proceso formativo de la persona. 
Los resultados obtenidos en el presente estudio permiten abordar el fenómeno de las experiencias positivas y negativas en parejas jóvenes y adolescentes desde una perspectiva multidimensional. Las relaciones se ven afectadas por un considerable número de variables sobre las cuales intervenir convenientemente, tratando de aportar herramientas y conocimientos dirigidos al aumento de los comportamientos saludables dentro de una relación de pareja más o menos estable. A la luz de los efectos positivos obtenidos tanto en el panorama internacional (Aldridge, Friedman, \& Gigans, 1993; Avery-Leaf et al., 1997; Bell \& Stanley, 2006; Levy, 1984; Foshee et al., 1998; Foshee et al., 2004; Rosenbluth, 2002; Schewe \& Anger, 2000; Wolfe et al., 2003, 2009) como dentro de nuestras fronteras (Díaz-Aguado \& Martínez Arias, 2002; Fernández-González，O’Leary，\& Muñoz-Rivas, 2013; Hernando-Gómez, 2007; Vizcarra et al., 2013;), los programas de prevención se erigen como pilares fundamentales para abordar las situaciones negativas en pareja, así como otras temáticas esenciales para el desarrollo adecuado de los adolescentes dentro de los centros educativos, en los que se llega a detectar diariamente situaciones que suponen el germen, en unos casos, o la propia constatación de la existencia de situaciones violentas entre ambos sexos en sus relaciones sentimentales y cotidianas.

Existen realidades a las que no podemos dar la espalda. Entre ellas destaca que los jóvenes mantienen relaciones de pareja en un alto porcentaje (en torno al $71 \%$ de la muestra); y que estas se inician, cada vez, en edades más tempranas (Price \& Byers, 1999), llegando a apreciar ciertas pautas comunes a estos primeros encuentros tales como la idealización del amor, la presencia de celos y un grado de control exagerado sobre la pareja (Soldevila et al., 2012), que puede llegar a confundir a los jóvenes respecto a la forma adecuada de amar o ser amado. A todo esto, debemos sumar la existencia de factores internalizantes y externalizantes que condicionan el transcurrir de dichas relaciones amorosas.

Desde el presente estudio, se deriva la necesidad de desarrollar programas dirigidos a toda la población escolar, con carácter permanente y de manera generalizada (prevención primaria) o específica (prevención secundaria), en función de los casos. La gran mayoría de las experiencias que se han realizado en centros escolares se han enfocado en aquellos colectivos más vulnerables sobre la posible aparición de situaciones negativas, así como en aquellos que tienen relaciones de pareja (principalmente adolescentes a partir de 14 años). Esto abre la posibilidad de poder ampliar el rango de acción a otras etapas o edades en las que, no habiéndose formado aún parejas, sí se pueda comenzar a aportar claves que logren desmontar mitos arraigados en los adolescentes y en la propia sociedad.

Estos programas han de contar con un número suficiente de sesiones que permitan un abordaje amplio y completo de aquellos condicionantes que influyen en la violencia en las parejas, ofreciendo al alumnado la posibilidad de realizar un acercamiento a esta realidad desde un planteamiento holístico, que favorezca su sensibilización y que les capacite suficientemente para detectar, analizar e intervenir adecuadamente en estos casos. Unido a esta ardua labor, desde una óptica sistémica (Hernando-Gómez, 2007), se debe incluir a las familias en estas acciones formativas; y también incentivar la participación del profesorado, como agente educativo de primer orden en la detección, intervención y asesoramiento al alumnado y sus familias. Estas actuaciones han de encontrar el respaldo de la 
comunidad educativa y el arraigo en los proyectos educativos y planes de estudio, para no correr el riesgo de quedar como mera actividad testimonial y recurrente, ligada a determinadas efemérides.

Esta intervención no ha de ceñirse a un colectivo concreto (por razón de edad, sexo, ideología, nivel sociocultural o económico), sino que el abordaje debe ser masivo a toda la población diana (adolescentes y jóvenes). Los resultados de nuestra investigación evidencian que, si bien es posible observar ciertas tendencias por sexos, en líneas generales existe una alta bidireccionalidad, ya puesta de manifiesto por otros autores (Fernández-González, 2013; Weisz et al., 2007). Lo que sí resultaría de gran interés, a la hora de diseñar actuaciones concretas, sería enfocar las intervenciones hacia aquellos tipos de experiencias negativas percibidas como más usuales por ambos sexos.
Por último, no se puede finalizar este artículo sin reflejar las limitaciones del estudio realizado. Entre estas se encuentran el método de selección de la muestra a través de procedimientos no probabilísticos (por accesibilidad). Asimismo, el carácter descriptivo y transversal del estudio imposibilita el establecimiento de relaciones de causalidad entre las variables personales contempladas y las experiencias vividas en parejas de jóvenes y adolescentes. Además, solo se han recogido las situaciones vividas, pero no se indaga por el impacto de esas situaciones. Por tanto, los resultados anteriores deben considerarse como una primera aproximación al estudio de las experiencias positivas y negativas vividas en el seno de las relaciones de parejas de jóvenes y adolescentes, esperamos seguir trabajando en esta línea para poder aportar datos que faciliten la intervención en este contexto.

\section{Referencias}

Achenbach, T. M. (1991). Manual for the Youth Self Report and 1991 profile. Burlington, Vermont: University of Vermont.

Alberdi, I. \& Rojas, L (2005). Violencia: tolerancia cero. Barcelona, España: Fundación La Caixa.

Aldridge, L., Friedman, C., \& Gigans, P. (1993). In touch with teens: A relationship violence prevention curriculum. Los Angeles, California: Los Angeles Commission on Assaults against Women.

Anderson, L. A. \& Whiston, S. C. (2005). Sexual assault education programs: Meta-analityc examination of their effectiveness. Psychology of Women Quarterly, 29(4), 374-388. http://dx.doi.org/10.1111/j.1471-6402.2005.00237.x

Arenas García, L. (2013). Sexismo en adolescentes y su implicación en la violencia de género. Boletín Criminológico, 144, 1-5. Recuperado de http://www.boletincriminologico.uma.es/boletines/144.pdf

Ashley, O. S. \& Foshee, V. A. (2005). Adolescent help-seeking for dating violence: Prevalence, sociodemographic correlates, and sources of help. Journal of Adolescent Health, $36(1), 25-31$.

http://dx.doi.org/10.1016/j.jadohealth.2003.12.014 
Avery-Leaf, S., Cascardi, M., O’Leary, K., \& Cano, A. (1997). Efficacy of a dating violence prevention program on attitudes justifying aggression. Journal of Adolescent Health, 21(5), 11-17. http://dx.doi.org/10.1016/S1054-139X(96)00309-6

Bell, J. \& Stanley, N. (2006). Learning about domestic violence: Young people's responses to a healthy relationships programme. Sex Education, 6(3), 237-250.

http://dx.doi.org/10.1080/14681810600836356

Centers for Disease Control and Prevention. (CDC, 2016). Understanding teen dating violence. Recuperado de https://www.cdc.gov/violenceprevention/pdf/teen-dating-violence-factsheet-a.pdf

Close, S. M. (2005). Dating violence prevention in middle school and high school youth. Journal of Child and Adolescent Psychiatric Nursing, 18(1), 2-9. http://dx.doi.org/10.1111/j.1744-6171.2005.00003.x

Cornelius, T. L. \& Resseguie, N. (2007). Primary and secondary prevention programs for dating violence: A review of the literature. Aggression and Violent Behavior, 12(3), 364375. http://dx.doi.org/10.1016/j.avb.2006.09.006

Corral, S. \& Calvete, E. (2006). Evaluación de la violencia en las relaciones de pareja mediante las escalas de tácticas para conflictos: estructura factorial y diferencias de género en jóvenes. Psicología Conductual, 14(2), 215-233. Recuperado de http://www.funveca.org/revista/pedidos/product.php?id_product=259

Crick, N. R. \& Grotpeter, J. K. (1995). Relational aggression, gender, and social psychological adjustment. Child Development, 66(3), 710-722. http://dx.doi.org/10.2307/1131945

Díaz-Aguado, M. J. \& Carvajal, I. (Dirs.) (2011). Igualdad y prevención de la violencia de género en la adolescencia y la juventud. Madrid, España: Ministerio de Sanidad, Igualdad y Servicios Sociales.

Díaz-Aguado, M. J. \& Martínez Arias, R. (2002). La construcción de la igualdad y la prevención de la violencia contra la mujer desde la educación secundaria (Serie Estudios, 73). Madrid, España: Instituto de la Mujer

Díaz-Aguado, M. J., Martínez-Arias, R., \& Martín-Babarro, J. (2013). La evolución de la adolescencia española sobre la igualdad y la prevención de la violencia de género. Madrid, España: Ministerio de Sanidad, Servicios Sociales e Igualdad.

Eaton, D. K., Kann, L., Kinchen, S., Ross, J., Hawkins, J., Harris, W. A., ... Wechsler, H. (2006). Youth risk behavior surveillance - United States, 2005. Morbidity and Mortality Weekly Report, 55(SS05), 1-108. Recuperado de https://www.cdc.gov/mmwr/preview/mmwrhtml/ss5505a1.htm

Eaton, D. K., Kann, L., Kinchen, S., Shanklin, S., Ross, J., Hawkins, J., ... Wechsler, H. (2010). Youth risk behavior surveillance - United States, 2009. Morbidity and Mortality Weekly Report, 59(SS05), 1-142. Recuperado de https://www.cdc.gov/mmwr/preview/mmwrhtml/ss5905a1.htm 
Fernández-Fuertes, A. A. \& Fuertes, A. (2005). Violencia sexual en las relaciones de pareja de los jóvenes. Sexología Integral, 2, 126-132.

Fernández-Fuertes, A. A. \& Fuertes, A. (2010). Physical and psychological aggression in dating relationships of Spanish adolescents: Motives and consequences. Child Abuse \& Neglect, 34(3), 183-191.

http://dx.doi.org/10.1016/j.chiabu.2010.01.002

Fernández-Fuertes, A., Fuertes, A., \& Pulido, R. (2006). Evaluación de la violencia en las relaciones de pareja de los adolescentes. Validación del Conflict in Adolescent Dating Relationships Inventory (CADRI) - versión española. International Journal of Clinical and Health Psychology, 6(2), 339-358. Recuperado de http://www.aepc.es/ijchp/articulos_pdf/ijchp-181.pdf

Fernández-Fuertes, A. A., Orgaz, B., \& Fuertes, A. (2011). Características del comportamiento agresivo en las parejas de los adolescentes españoles. Psicología Conductual, 19(3), 501-522. Recuperado de http://www.funveca.org/revista/pedidos/product.php?id_product=505

Fernández-González, L. (2013). Prevención de la violencia en las relaciones de noviazgo: aplicación y valoración de un programa para adolescentes (Tesis doctoral, Universidad Autónoma de Madrid, España). Recuperada de https://is.gd/rHhXR1

Fernández-González, L., O’Leary, K. D., \& Muñoz-Rivas, M. J. (2013). We are not joking: Need for controls in reports of dating violence. Journal of Interpersonal Violence, 28(3), 602-620. http://dx.doi.org/10.1177/0886260512455518

Ferreira, G. B. (1992). Hombres violentos, mujeres maltratadas: aportes a la investigación y tratamiento de un problema social. Buenos Aires, Argentina: Editorial Sudamericana.

Follingstad, D. R., Bradley, R. G., Laughlin, J. E., \& Burke, L. (1999). Risk factors and correlates of dating violence: The relevance of examining frequency and severity levels in a college sample. Violence and Victims, 14(4), 365-380. Recuperado de https://www.ncbi.nlm.nih.gov/pubmed/10751045

Foshee, V. A., Bauman, K. E., Arriaga, X. B., Helms, R. W., Koch, G. G., \& Linder, G. F. (1998). An evaluation of safe dates, an adolescent dating violence prevention program. American Journal of Public Health, 88(1), 45-50. http://dx.doi.org/10.2105/AJPH.88.1.45

Foshee, V. A., Bauman, K. E., Ennett, S. T., Linder, G. F., Benefield, T., \& Suchindran, C. (2004). Assessing the long-term effects of the safe dates program and booster in preventing and reducting adolescent dating violence victimization and perpetration. American Journal of Public Health, 94(4), 619-625. http://dx.doi.org/10.2105/AJPH.94.4.619

Foshee, V. A., Reyes, H. L. M., Ennett, S. T., Suchindran, C., Mathias, J. P., Karriker-Jaffe, K. J., Bauman, K. E., \& Benefield, T. S. (2011). Risk and protective factors distinguishing profiles of adolescent peer and dating violence perpetration. Journal of Adolescent Health, 48(4), 344-350.

http://dx.doi.org/10.1016/j.jadohealth.2010.07.030 
Garaigordobil, M., Aliri, J., \& Martínez-Valderrey, V. (2013). Justificación de la violencia durante la adolescencia: diferencias en función de variables sociodemográficas. European Journal of Education \& Psychology, 6(2), 83-93. Recuperado de https://dialnet.unirioja.es/descarga/articulo/4832388.pdf

García Díaz, V., Fernández Feito, A., Rodríguez Díaz, F. J., López González, M. L., Mosteiro Díaz, M. D. P., \& Lana Pérez, A. (2013). Violencia de género en estudiantes de enfermería durante sus relaciones de noviazgo. Atención Primaria, 45(6), 290-296. http://dx.doi.org/10.1016/j.aprim.2012.11.013

Gomez, A. M., Speizer, I. S., \& Moracco, K. E. (2011). Linkages between gender equity and intimate partner violence among urban Brazilian youth. Journal of Adolescent Health, 49(4), 393-399. http://dx.doi.org/10.1016/j.jadohealth.2011.01.016

González Méndez, R. \& Santana Hernández, J. D. (2001). La violencia en parejas jóvenes. Psicothema, 13(1), 127-131. Recuperado de http://www.psicothema.com/pdf/423.pdf

González-Ortega, I., Echeburúa, E., \& de Corral, P. (2008). Variables significativas en las relaciones violentas en parejas jóvenes: una revisión. Behavioral Psychology / Psicología Conductual, 16(2), 207-225. Recuperado de http://www.ehu.eus/echeburua/pdfs/04GONZALEZ.pdf

Gorrotxategi, M. \& De Haro, I. M. (1999). Materiales didácticos para la prevención de la violencia de género. Educación secundaria. Málaga, España: Consejería de Educación y Ciencia. Junta de Andalucía.

Greytak, E. A. (2003). Educating for the prevention of sexual abuse: An investigation of school-based programs for high school students and their applicability to urban schools. Penn GSE Perspectives on Urban Education, 2(1), 1-15. Recuperado de http://www.urbanedjournal.org/printpdf/139

Health Canada. (1995). Dating violence. Canada: National Clearinghouse on Family Violence.

Hernando-Gómez, A. (2007). La prevención de la violencia de género en adolescentes. Una experiencia en el ámbito educativo. Apuntes de Psicología 25(3), 325-340. Recuperado de http://www.uhu.es/angel.hernando/documentos/2007_Apuntes_de_Psicologia.pdf

Karakurt, G. \& Cumbie, T. (2012). The relationship between egalitarianism, dominance, and violence in intimate relationships. Journal of Family Violence, 27(2), 115-122. http://dx.doi.org/10.1007/s10896-011-9408-y

Kaukinen, C., Gover, A. R., \& Hartman, J. L. (2012). College women's experiences of dating violence in casual and exclusive relationships. American Journal of Criminal Justice, 37(7), 146-162. http://dx.doi.org/10.1007/s12103-011-9113-7

Lemos Giráldez, S., Vallejo Seco, G., \& Sandoval Mena, M. (2002). Estructura factorial del Youth Self Report (YSR). Psicothema, 14(4), 816-822. Recuperado de http://www.psicothema.com/psicothema.asp?id=804 
Levy, B. (1984). Skills for violence free relationships: Curriculum for young people ages $13-$ 18. Saint Paul, Minnesota: Minnesota Coalition for Battered Women.

Makepeace, J. M. (1981). Courtship violence among college students. Family Relations, 30(1), 97-102.

http://dx.doi.org/10.2307/584242

Menesini, E. \& Nocentini, A. (2008). Comportamenti aggressivi nelle prime esperienze sentimentali in adolescenza. Giornale Italiano di Psicologia, 35(2), 407-434. http://dx.doi.org/10.1421/27217

Meras Lliebre, A. (2003). Prevención de la violencia de género en adolescentes. Estudios de Juventud, 62(3), 143-150. Recuperado de http://www.injuve.es/sites/default/files/art11.pdf

Molidor, C. \& Tolman, R. M. (1998). Gender and contextual factor in adolescent dating violence. Violence against Women, 4(2), 180-194. http://dx.doi.org/10.1177/1077801298004002004

Muñoz-Rivas, M. J., Graña, J. L., O’Leary, K. D., \& González, M. P. (2007). Aggression in adolescent dating relationships: Prevalence, justification, and health consequences. Journal of Adolescent Health, 40(4), 298-304.

http://dx.doi.org/10.1016/j.jadohealth.2006.11.137

Muñoz-Rivas, M. J., Graña, J. L., O’Leary, K. D., \& González, M. P. (2009). Prevalence and predictors of sexual aggression in dating relationships of adolescents and young adults. Psicothema, 21(2), 234-240. Recuperado de http://www.psicothema.com/psicothema.asp?id=3620

Ocampo, B. W., Shelley, G. A., \& Jaycox, L. H. (2007). Latino teens talk about help-seeking and help-giving in relation to dating violence. Violence against Women, 13(2), 172-189. http://dx.doi.org/10.1177/1077801206296982

O'Leary, K. D. \& Slep, A. M. S. (2012). Prevention of partner violence by focusing on behaviors of both young males and females. Prevention Science, 13(4), 329-339. http://dx.doi.org/10.1007/s11121-011-0237-2

O’Leary, K. D., Slep, A. M. S., Avery-Leaf, S., \& Cascardi, M. (2008). Gender differences in dating aggression among multiethnic high school students. Journal of Adolescent Health, 42(5), 473-479. http://dx.doi.org/10.1016/j.jadohealth.2007.09.012

Organización Mundial de la Salud, OMS (2000). Informe sobre la salud en el Mundo 2000: Mejorar el desempeño de los sistemas de salud. Ginebra, Suiza: Organización Mundial de la Salud.

Ortega, R., Ortega Rivera, F. J., \& Sánchez, V. (2008). Violencia sexual entre compañeros y violencia en parejas adolescentes. International Journal of Psychology and Psychological Therapy, 8(1), 63-72. Recuperado de http://www.redalyc.org/pdf/560/56080106.pdf

Pazos Gómez, M., Oliva Delgado, A., \& Hernando-Gómez, A. (2014). Violencia en relaciones de parejas jóvenes y adolescentes. Revista Latinoamericana de Psicología, 46(3). 148-159. http://dx.doi.org/10.1016/S0120-0534(14)70018-4 
Perles Novas, F., San Martín García, J., Canto Ortiz, J., \& Moreno Jiménez, P. (2011). Inteligencia emocional, celos, tendencia al abuso y estrategias de resolución de conflicto en la pareja. Escritos de Psicología, 4(1), 34-43.

http://dx.doi.org/10.5231/psy.writ.2011.0605

Price, E. L. \& Byers, E. S. (1999).The attitudes towards dating violence scales: Development and initial validation. Journal of Family Violence, 14(4), 351-375.

http://dx.doi.org/10.1023/A:1022830114772

Roscoe, B. \& Callahan, J. E. (1985). Adolescents' self-report of violence in families and dating relations. Adolescence, 20(79), 545-554. Recuperado de

https://is.gd/6HcpDU

Rosenbluth, B. (2002). Expect respect: A school based program promoting health relationships for youth. Harrisburg, Pennsylvania: National Resource Center on Domestic Violence.

Rubio-Garay, F., Carrasco, M. A., Amor, P. J., \& López-González, M. A. (2015). Factores asociados a la violencia en el noviazgo entre adolescentes: una revisión crítica. Anuario de Psicología Jurídica, 25(1), 47-56.

http://dx.doi.org/10.1016/j.apj.2015.01.001

Schewe, P. A. \& Anger, I. (2000). Southside teens about respect (STAR): An intervention to promote healthy relationships and prevent teen dating violence. Trabajo presentado en la Conferencia Nacional de Prevención Sexual, Dallas, Texas.

Sears, H. A., Byers, E. S., \& Price, E. L. (2007). The co-occurrence of adolescent boys' and girls' use of psychologically, physically, and sexually abusive behaviours in their dating relationships. Journal of Adolescence, 30(3), 487-504.

http://dx.doi.org/10.1016/j.adolescence.2006.05.002

Soldevila, A., Domínguez, A., Giordano, R., Fuentes, S., \& Consolini, L. (2012). ¿Celos, amor, culpa o patología? Cómo perciben la violencia de género en sus relaciones de pareja los/as estudiantes de Trabajo Social. En Actas del $2^{\circ}$ Congreso Interdisciplinario sobre Género y Sociedad: 'Lo personal es político', 1(1). Córdoba, Argentina: Universidad Nacional de Córdoba. Recuperado de

http://www.ffyh.unc.edu.ar/piemg/noticias/ii-congreso-2012-actas.html

Straus, M. A. (2004). Prevalence of violence against dating partners by male and female university students worldwide. Violence against Women, 10(7), 790-811. http://dx.doi.org/10.1177/1077801204265552

Straus, M. A. \& Ramirez, I. L. (2007). Gender symmetry in prevalence, severity, and chronicity of physical aggression against dating partners by university students in Mexico and USA. Aggressive Behavior, 33(4), 281-290.

http://dx.doi.org/10.1002/ab.20199

Sugarman, D. B. \& Hotaling, G. T. (1989). Dating violence: Prevalence, context, and risk markers. En M. A. Pirog-Good \& J. E. Stets (Eds.), Violence in dating relationships: Emerging social issues (pp. 3-32). New York, New York: Praeger. 
Tontodonato, P. \& Crew, B. K. (1992). Dating violence, social learning theory, and gender: A multivariate analysis. Violence and Victims, 7(1), 3-14. Recuperado de https://is.gd/i2gt7M

Torres, C, Robles, J. M., \& De Marco, S. (2013). El ciberacoso como forma de ejercer la violencia de género en la juventud: un riesgo en la sociedad de la información y el conocimiento. Madrid, España: Servicio de publicaciones del Ministerio de Sanidad, Servicios Sociales e Igualdad.

Vizcarra Larrañaga, M. B. \& Poo Figueroa, A. M. (2011). Violencia de pareja en estudiantes universitarios del sur de Chile. Universitas Psychologica, 10(1), 89-98. Recuperado de http://www.redalyc.org/articulo.oa?id=64719284008

Vizcarra, M. B., Poo, A. M., \& Donoso, T. (2013). Programa educativo para la prevención de la violencia en el noviazgo. Revista de Psicología, 22(1), 48-61.

http://dx.doi.org/10.5354/0719-0581.2013.27719

Weisz, A. N., Tolman, R. M., Callahan M. R., Saunders, D. G., \& Black, B. M. (2007). Informal helpers' responses when adolescents tell them about dating violence or romantic relationship problems. Journal of Adolescence, 30(5), 853-868.

http://dx.doi.org/10.1016/j.adolescence.2006.09.004

Wolfe, D. A., Crooks, C., Jaffe, P., Chiodo, D., Hughes, R., Ellis, W., Stitt, L., \& Donner, A. (2009). A school-based program to prevent adolescent dating violence: A cluster randomized trial. Archives of Pediatrics \& Adolescent Medicine, 163(8), 692-699. http://dx.doi.org/10.1001/archpediatrics.2009.69

Wolfe, D. A., Wekerle, C., Gough, R., \& Reitzel-Jaffe, D. (1996). The youth relationships manual: A group approach with adolescents for the prevention of woman abuse and the promotion of healthy relationships. Thousand Oaks, California: Sage Publications.

Wolfe, D. A., Wekerle, C., Scott, K., Straatman, A., Grasley, C., \& Reitzel-Jaffe, D. (2003). Dating violence prevention with at-risk youth: A controlled outcome evaluation. Journal of Consulting and Clinical Psychology, 71(2), 279-291. http://dx.doi.org/10.1037/0022-006X.71.2.279 